\title{
Simulation and Experimental Validation of Gain-Control Parallel Hybrid Fiber Amplifier
}

\author{
Mudhafar Hussein Ali ${ }^{1}$, Fairuz Abdullah ${ }^{1 * *}$, Md Zaini Jamaludin ${ }^{1}$, Mohammed Hayder \\ Al-Mansoori $^{2}$, Thamer Fahad Al-Mashhadani ${ }^{1}$, and Abdulla Khudiar Abass ${ }^{1}$ \\ ${ }^{l}$ Center for Photonic Technologies, College of Engineering, Universiti Tenaga Nasional, Jalan \\ IKRAM-UNITEN, 43000 Kajang, Selangor, Malaysia \\ ${ }^{2}$ Faculty of Engineering, Sohar University, PO Box 44, Sohar, PCI 311, Oman
}

(Received July 17, 2014 : revised October 8, 2014 : accepted November 10, 2014)

\begin{abstract}
We demonstrate a simulation of a parallel hybrid fiber amplifier in the $\mathrm{C}+\mathrm{L}$-band with a gain controlling technique. A variable optical coupler is used to control the input signal power for both EDFA and RFA branches. The gain spectra of the $\mathrm{C}+\mathrm{L}-$ band are flattened by optimizing the coupling ratio of the input signal power. In order to enhance the pump conversion efficiency, the EDFA branch was pumped by the residual Raman pump power. A gain bandwidth of $60 \mathrm{~nm}$ from $1530 \mathrm{~nm}$ to $1590 \mathrm{~nm}$ is obtained with large input signal power less than $-5 \mathrm{dBm}$. The gain variation is about $1.06 \mathrm{~dB}$ at a small input signal power of $-30 \mathrm{dBm}$, and it is reduced to $0.77 \mathrm{~dB}$ at the large input signal power of $-5 \mathrm{dBm}$. The experimental results show close agreement with the simulation results.
Keywords : Parallel hybrid fiber amplifier, Raman fiber amplifier, Erbium doped fiber amplifier, Gain control
OCIS codes : (060.4370) Nonlinear optics, fibers; (060.2410) Fibers, erbium; (060.2320) Fiberoptics amplifier and oscillators

\section{INTRODUCTION}

Hybrid Raman/erbium-doped fiber amplifiers are an enabling and promising technology for future dense wavelength-divisionmultiplexing (DWDM) multi-terabit systems, as it has been shown by recent experimental results [1-7]. Hybrid fiber amplifiers are designed to maximize the span length, minimize the impairments caused by fiber nonlinearities, enhance erbium doped fiber amplifier (EDFA) bandwidth, provide sufficient increase in overall signal gain and enhance the pump conversion efficiency [8-11]. Two main configurations were used in the design of the hybrid fiber amplifiers; serial hybrid fiber amplifiers (S-HFA) and parallel hybrid fiber amplifiers (P-HFA).

In serial architecture, the input signal has two stages of amplification based on a single path, In other words, the output signal of the first stage is used as an input signal for the second stage. Even though this type of amplifier has high overall gain and an acceptable noise figure, there is still an issue in gain flatness. Masuda et. al. in 1998 reported a wide 3-dB gain-bandwidth hybrid fiber amplifier without using any gain-equalizer to produce a flat gain spectrum for a $76 \mathrm{~nm}$ range from $1531.5 \mathrm{~nm}$ to $1607.5 \mathrm{~nm}$. However, the design used three amplification stages and five laser diodes as pumping sources, one unit to pump the EDFA and four units with different operating wavelengths to pump the Raman fiber amplifier (RFA) [12].

In 2008, Liang et. al. reported an S-HFA with wide (65 $\mathrm{nm})$ amplification bandwidth from $1530 \mathrm{~nm}$ to $1595 \mathrm{~nm}$ and lower gain variation $(<0.2 \mathrm{~dB})$ [13]. However, to get the low gain variation, a complicated array of fiber Bragg grating mirrors was employed.

The P-HFA in which the input signal was separated into two wavelength bands (C and $\mathrm{L}$ ) was reported in [14-16]. One method used to improve gain flatness is to split pump power going into gain media. The optimum ratio of pump power going to erbium and Raman was found to be 1:29. The reported gain is $3-\mathrm{dB}$ at input signal power of -20 $\mathrm{dBm}$. However, as the input power increases, the gain variation degraded to 4 and $6 \mathrm{~dB}$ at input signal power of $-10 \mathrm{dBm}$ and $0 \mathrm{dBm}$, respectively.

In this paper, we proposed a new P-HFA concept where, instead of dividing the pump power, the input signal is

\footnotetext{
*Corresponding author: fairuz@ieee.org

Color versions of one or more of the figures in this paper are available online.
} 
divided into two. The gain spectrum flatness is controlled by varying the input signal ratio. Wide flat gain bandwidth over a range of $60 \mathrm{~nm}$ from $1530 \mathrm{~nm}$ to $1590 \mathrm{~nm}$ was obtained at input signal power range from $-30 \mathrm{dBm}$ to $-5 \mathrm{dBm}$.

\section{GAIN AND NOISE FIGURE OF PARALLEL HYBRID FIBER AMPLIFIER}

Figure 1 shows the power tracing of the light path for the proposed P-HFA. The VOC is used to control the input signal power ratio. In this equivalent light path diagram, the power tracing is adopted to calculate the overall hybrid gain.

The hybrid gain $\mathrm{G}_{\mathrm{H}}$ is given by:

$$
\begin{aligned}
& G_{H}=\frac{P_{\text {out }}}{P_{\text {in }}} \\
& P_{\text {out }}(m W)=\left[n P_{\text {in }} G_{E}+(1-n) P_{\text {in }} G_{R}\right] * \alpha_{c}
\end{aligned}
$$

where, $0<n<1$ represents the coupling ratio of VOC, $P_{\text {in }}(\mathrm{mW})$ is the input signal power, $P_{\text {out }}(\mathrm{mW})$ is the output signal power, $G_{E}$ and $G_{R}$ are the gain factor of the EDFA and RFA, respectively and $\alpha_{C}$ is the OFC loss factor.

Thus, the P-HFA gain factor as a function of signal wavelength $\lambda$ is:

$$
G_{H}(\lambda)=\left[n G_{E}(\lambda)+(1-n) G_{R}(\lambda)\right] * \alpha_{c}
$$

$G_{E}$ in Eq.(3) is based on a derived model presented by [17]. Three fiber parameters were used in this proposed model which are: absorption coefficient $\left(\alpha_{k}\right)$, gain coefficient $\left(g_{k}\right)$, and a fiber saturation parameter $(\zeta)$. These parameters are obtained by conventional fiber measurement techniques covered by [17]. The saturation parameter can be defined theoretically as:

$$
\zeta=\frac{\pi b_{e f f}^{2} n_{t}}{\tau}
$$

where: $b_{\text {eff }}$ is the equivalent radius of the doped region, $n_{t}$ is the local erbium ion density and $\tau$ is the metastable lifetime parameter.

The absorption and gain coefficients are expressed in terms of distributions of the ions and optical modes:

$$
\begin{aligned}
& \alpha_{k}\left(\lambda_{k}\right)=\sigma_{a}\left(\lambda_{k}\right) \int_{0}^{2 \pi} \int_{0}^{\infty} i_{k}(r, \phi) n_{t}(r, \phi, z) r \quad d r d \phi \\
& g_{k}\left(\lambda_{k}\right)=\sigma_{e}\left(\lambda_{k}\right) \int_{0}^{2 \pi} \int_{0}^{\infty} i_{k}(r, \phi) n_{t}(r, \phi, z) r \quad d r d \phi
\end{aligned}
$$

where, $\sigma_{c}\left(\lambda_{k}\right)$ and $\sigma_{e}\left(\lambda_{k}\right)$ are the absorption and emission cross-section of the $k^{\text {th }}$ beam and $i_{k}(r, \phi)$ is the normalized optical intensity.

For a uniform ion distribution, the absorption and gain coefficients can be simplified as:

$$
\begin{aligned}
& \alpha_{k}\left(\lambda_{k}\right)=\Gamma_{k}\left(\lambda_{k}\right) \cdot \bar{n}_{t} \cdot \sigma_{a}\left(\lambda_{k}\right) \\
& g_{k}\left(\lambda_{k}\right)=\Gamma_{k}\left(\lambda_{k}\right) \cdot \bar{n}_{t} \cdot \sigma_{e}\left(\lambda_{k}\right)
\end{aligned}
$$

where: $\Gamma_{k}\left(\lambda_{k}\right)$ is the overlap integral.

The propagation equation in terms of saturation parameter, and absorption and emission coefficients in [17]:

$$
\begin{aligned}
\frac{d P_{k}(z)}{d z} & =u_{k} \cdot P_{k}(z) \cdot\left[g_{k}\left(\left(v_{k}\right)+\alpha_{k}\left(v_{k}\right)\right) \cdot \frac{\bar{n}_{2}}{\bar{n}_{t}}-\alpha_{k}\left(v_{k}\right)-l_{k}\right] \\
& +u_{k} \cdot P_{o k} \cdot g_{k}\left(v_{k}\right) \frac{\bar{n}_{2}}{\bar{n}_{t}}
\end{aligned}
$$

where each beam propagates in the forward $u_{k}=1$ or backward $u_{k}=-1$ direction, and the spontaneous emission contribution from the local metastable population $\overline{n_{2}}$ and $l_{k}$ is the background loss. The steady-state solution of the rate equation can be rewritten as:

$$
\frac{\bar{n}_{2}}{\bar{n}_{t}}(z)=\frac{\sum_{k=1}^{n} \frac{P_{k}(z) \cdot \alpha_{k} v_{k}}{h \cdot v_{k} \cdot \zeta}}{1+\sum_{k=1}^{n} \frac{P_{k}(z) \cdot \alpha_{k}\left(v_{k}\right)+g_{k}\left(v_{k}\right)}{h \cdot v_{k} \cdot \zeta}}
$$

Finally $G_{E}$ is presented as the ratio of the output signal power to the input signal power [18]:

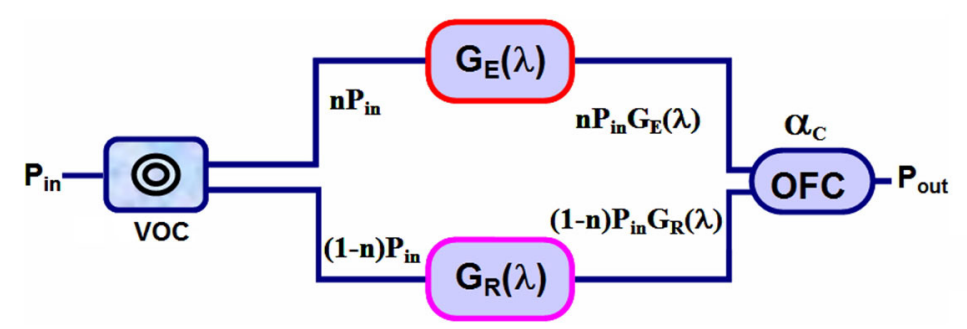

FIG. 1. Equivalent light path of PHFA. VOC: Variable Optical Coupler, OFC: Optical Fiber Coupler. 


$$
G_{E}=\frac{P(L)}{P(0)}
$$

where: $\mathrm{P}(0)$ represent the input signal power and $\mathrm{P}(\mathrm{L})$ is the output signal power obtained by solving Eq.(10) under the homogeneous line broadening case.

In addition, $G_{R}$ in Eq. (3) is obtained by solving the output signal power equation using the numerical solution presented by [19]:

$$
\begin{aligned}
& \frac{d P_{f}\left(z, v_{i}\right)}{d z}=-\alpha\left(v_{i}\right) P_{f}\left(z, v_{i}\right)+\gamma\left(v_{i}\right) P_{b}\left(z, v_{i}\right)+P_{f}\left(z, v_{i}\right) \sum_{v_{i}<\zeta} \frac{g_{R}\left(v_{i}-v_{j}\right)}{K_{e f f} A_{e f f}} \\
& {\left[P_{f}\left(z, v_{i}\right)+P_{b}\left(z, v_{j}\right)\right]+h \Delta v_{i} \sum_{v_{i}<v_{j}} \frac{g_{R}\left(v_{i}-v_{j}\right)}{A_{e f f}}\left[P_{f}\left(z, v_{j}\right)+P_{b}\left(z, v_{j}\right)\right][1+} \\
& \left.\left(\exp \left[h \frac{\left(v_{i}-v_{j}\right)}{k T}\right]-1\right)^{-1}\right]-P_{f}\left(z, v_{i}\right) \sum_{v_{i}>v_{j}} \frac{g_{R}\left(v_{i}-v_{j}\right)}{K_{e f f} A e f f} \frac{v_{i}}{v_{j}}\left[P_{f}\left(z, v_{j}\right)\right. \\
& \left.+P_{b}\left(z, v_{j}\right)\right]-2 h v_{i} \Delta v_{i} P_{f}\left(z, v_{i}\right) \sum_{v_{i}>v_{i}} \frac{g_{R}\left(v_{i}-v_{j}\right)}{A_{e f f}}\left[1+\left(\exp \left[h \frac{\left(v_{i}-v_{j}\right)}{k T}\right]-1\right)^{-1}\right]
\end{aligned}
$$

where $v_{i}$ and $v_{j}$ are frequencies, $g_{R}$ is the Raman gain coefficient, $\alpha(v)$ is the fiber attenuation, $\gamma(v)$ is the Rayleigh backscattering coefficient, $g_{R}\left(v_{i}-v_{j}\right)$ is the Raman gain coefficient for frequency difference $\left(v_{i}-v_{j}\right), P_{b}\left(z, v_{i}\right)$ is the backward propagating power including sampled, parameterized, and noise bins signals, $A_{\text {eff }}$ is the effective core area, $K_{\text {eff }}$ is the polarization factor, $\Delta v$ is the frequency interval, $h$ is Plank's constant, $k$ is the Boltzmann's constant and $T$ is the absolute temperature.

Raman gain reference pump and the Raman peak gain are needed in the simulation. Therefore, these two values were obtained by solving the following formula derived by [19].

$$
g_{R}=\frac{P_{R}}{\lambda_{P}} g_{N}
$$

where, $P_{R}$ is the Raman gain peak, $\lambda_{P}$ is the gain reference pump and $g_{N}$ is the normalized Raman gain. Finally, $G_{R}$ was calculated as the ratio of the output signal power to the input signal power [20]:

$$
G_{R}=\frac{P(L)}{P(0)}
$$

where $P(0)$ is the input signal power and $\mathrm{P}(\mathrm{L})$ represents the output signal power obtained form Eq.(12).

In terms of the NF of P-HFA, the EDFA NF was presented by [18], given as:

$$
N F_{E}(d B)=10 \log \frac{P_{A S E}}{h v G_{E} B_{o}}+\frac{1}{G_{E}}
$$

where $P_{A S E}$ is the generated noise in EDFA, $h$ is Planck's constant, $V$ is the optical frequency of the input signal in $\mathrm{Hz}$,
$G_{E}$ is the gain of the EDFA and $B_{o}$ is the optical bandwidth in $\mathrm{Hz}$.

The NF in RFA can be simply estimated by calculating the Raman gain $G_{R}$ and the $P_{A S E}$ power [21]:

$$
N F(d B)=10 \log \frac{2 P_{A S E}}{h v G_{R} B_{o}}+\frac{1}{G_{R}}
$$

where $P_{A S E}$ and $G_{R}$ represent the generated noise and the gain in the RFA respectively.

The summation of Eqns. (15) and (16) can represent the hybrid gain because the signal is split into two identical source (in term of $\lambda$ ) to be amplified by different gain media.

$$
N F(d B)_{P-H F A}=N F(d B)_{E}+N F(d B)_{R}-\alpha_{c}(d B)
$$

\section{SIMULATION DESIGN}

In the simulation design the bi-directional fiber model is adopted as a Raman gain medium. In this model, pumpto-pump, signal-to-signal and pump-to-signal Raman interactions, spontaneous Raman emission and its temperature dependency and stimulated Raman scattering (SRS) are considered. In addition, the pump depletions due to Raman energy transfer, high-order Stokes generation, multiple Rayleigh backscattering, fiber loss and spontaneous emission noise were also considered. Moreover, the stimulated Brillouin scattering (SBS) and pump depletions due to SRS effects are included in this model $[22,23]$.

\subsection{Simulation Setup}

Figure 2 shows the design of P-HFA. A variable optical coupler is used to control the input signal power between the two branches (EDFA and RFA). The input signal is provided by a tunable laser source (TLS) with power range from -30 to $-5 \mathrm{dBm}$ at wavelength range from 1530 $\mathrm{nm}$ to $1595 \mathrm{~nm}$ with linewidth of $150 \mathrm{kHz}$. The RFA is a $7 \mathrm{~km}$ of dispersion compensating fiber (DCF), pumped in a counter pump direction by a Raman pump unit (RPU) with output power of $800 \mathrm{~mW}$ at $1480 \mathrm{~nm}$. The DCF has total loss of $4.4 \mathrm{~dB}$, effective area of $18.5 \mu \mathrm{m}^{2}$, nonlinear coefficient of $14.5 \times 10^{-10} \mathrm{~W}^{-1}$ and dispersion parameter of $-110 \mathrm{ps} / \mathrm{nm} / \mathrm{km}$. The EDFA is a $3 \mathrm{~m}$ erbium doped fiber (EDF) pumped by the residual Raman pump power about $75 \mathrm{~mW}$, controlled by a variable optical attenuator (VOA).

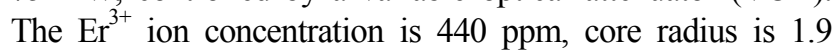
$\mu \mathrm{m}, \mathrm{Er}$ doping radius is $1.9 \mu \mathrm{m}$, and cut-off wavelength is $1300 \mathrm{~nm}$. A wavelength selective coupler (WSC) is used to separate the residual Raman pump from the reflected Rayleigh scattering signal. An optical fiber coupler (OFC) is used to collect the output signal from the two branches. Finally, two optical spectrum analyzers are used, the OSA1 to record the total system gain and OSA2 to record the Brillouin Stokes power. 


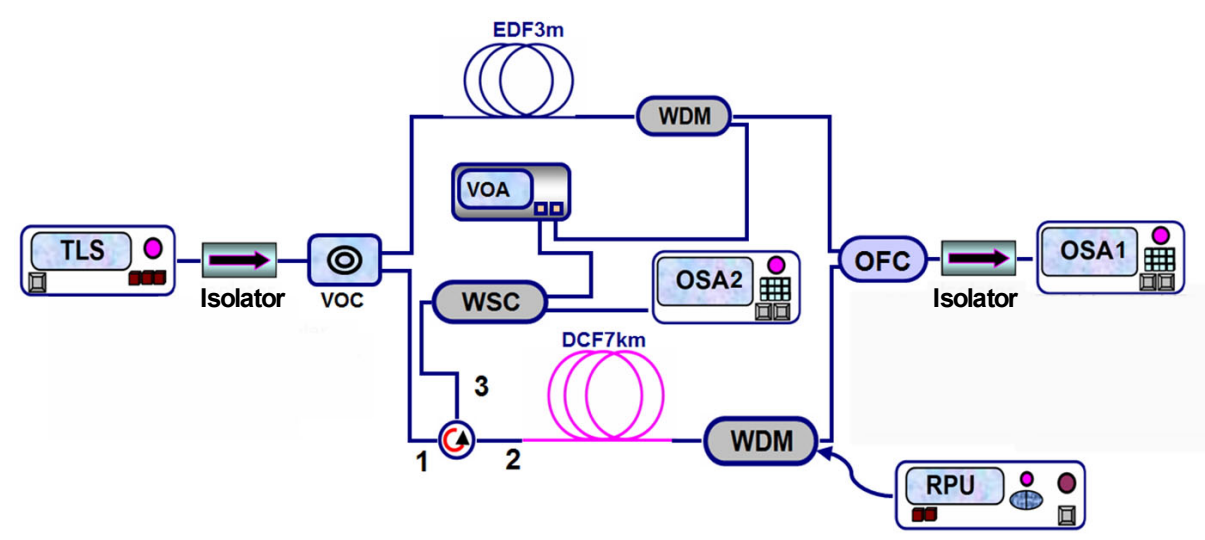

FIG. 2. Schematic diagram of parallel hybrid utilizing gain controlled technique. TLS: Tunable Laser Source, VOC: Variable Optical Coupler, VOA: Variable Optical Attenuator, OSA: Optical Spectrum Analyzer, RPU: Raman Pump Unit, WDM: Wavelength Division Multiplexer, WSC: Wavelength Selector Coupler, DCF: Dispersion Compensating Fiber, EDF: Erbium Doped Fiber, OFC: Optical Fiber Coupler.

\subsection{Simulation Results}

The overall gain profile of different coupling ratios with $P_{\text {in }}$ of $-30 \mathrm{dBm}$ and $-5 \mathrm{dBm}$ is depicted in Fig. 3 (a) and (b), respectively. The hybrid gain values are calculated using

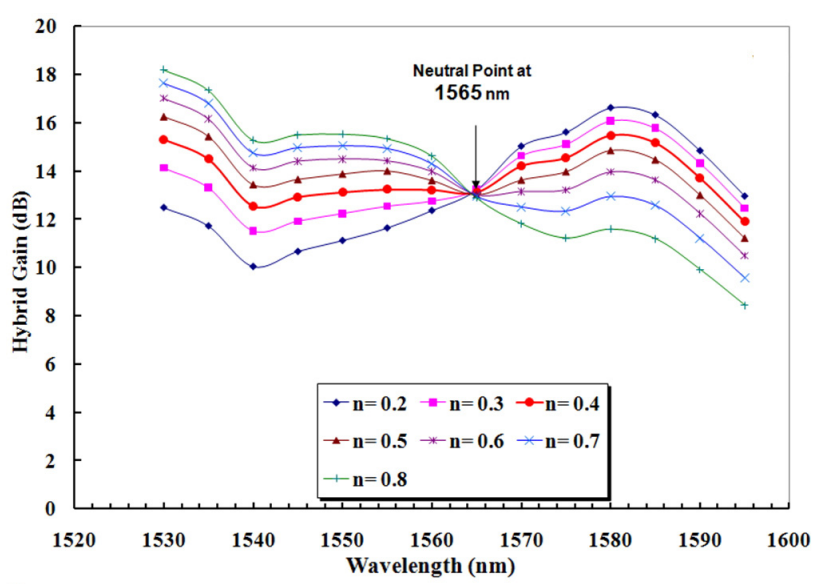

(a)

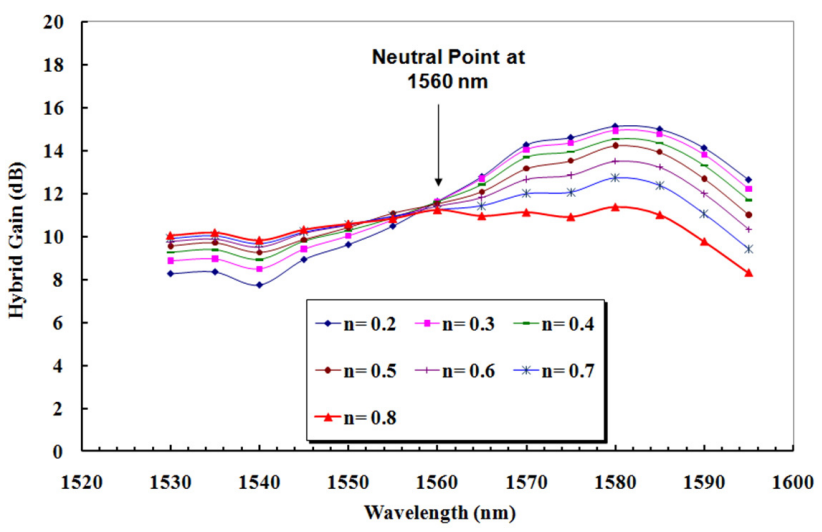

(b)

FIG. 3. Hybrid gain profile at different coupling ratios for: (a) $P_{\text {in }}$ of $-30 \mathrm{dBm}$ and (b) $\mathrm{P}_{\text {in }}$ of $-5 \mathrm{dBm}$.
Eq. (4). The coupling ratio $n$ represents the percentage of input signal power that goes into EDFA.

The overall gain spectra can be divided into three regions: 1) C-band region where EDFA is more effective, 2) L-band region in which the RFA is more efficient and 3) neutral region or balance point where the input signal is amplified by equal gain from both EDFA and RFA for all coupling ratios.

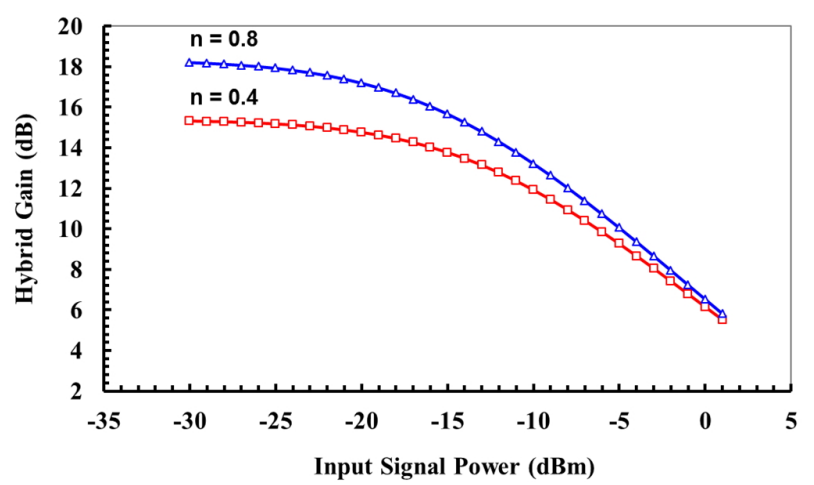

(a)

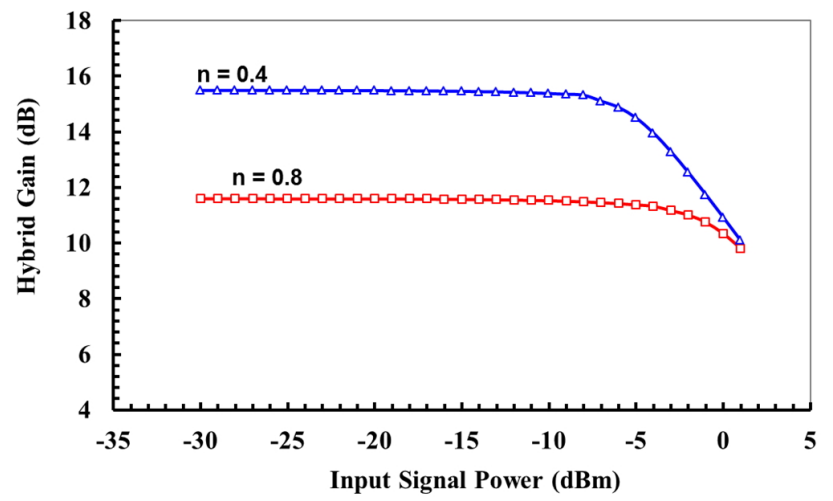

(b)

FIG. 4. Hybrid gain vs. $\mathrm{P}_{\text {in }}$ for two different wavelengths (a) $1530 \mathrm{~nm}$ and (b) $1580 \mathrm{~nm}$. 
For small $P_{\text {in }}(-30 \mathrm{dBm})$, as the $n$ value changes from 0.2 to 0.8 , the gain shows decreasing trend from $1525 \mathrm{~nm}$ to neutral point $(1565 \mathrm{~nm})$ and increasing trend from there on until $1595 \mathrm{~nm}$. At $n=0.4$, the gain variation is smallest compared to others. For large input $(-5 \mathrm{dBm})$, as depicted in Fig. 3(b), the trend is similar but the neutral point is slightly shifted to $1560 \mathrm{~nm}$. The best gain variation is when $n=0.8$.

At small input signal, no saturation occurs in RFA for both $n$ values while the EDFA starts to saturate at signal power above $-15 \mathrm{dBm}$ causing the hybrid gain to decrease (Refer Fig. 4). As a result to this saturation effect in the EDFA, an increment in $n$ factor is required to increase the C-band gain level and produce wide gain flatness.

\section{EXPERIMENTAL VALIDATION}

The simulation was validated by experiment for two values of $n, 0.4$ and 0.8 since they give the best gain flatness for small and large signal, respectively. The hybrid gain spectra are illustrated in Fig. 5. The experimental result shows very close agreement with the simulation where the gain average obtained experimentally is $13.3 \mathrm{~dB}$ for small

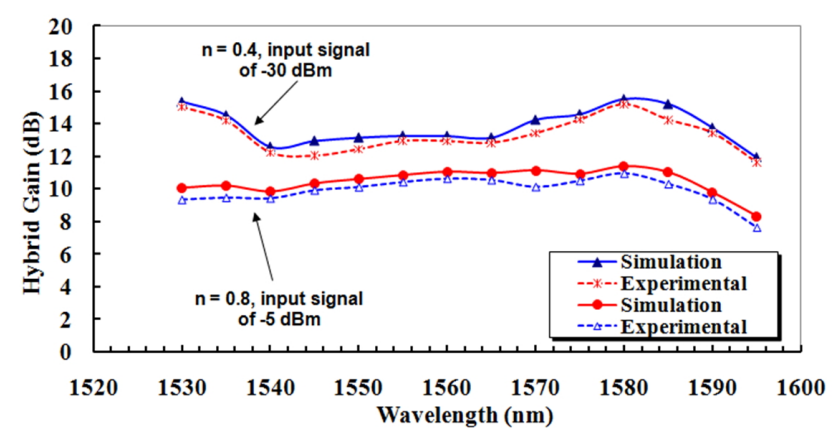

FIG. 5. Simulation \& experimental validation of hybrid gain spectra for two coupling ratios of $n=0.4$ at the $P_{\text {in }}$ of $-30 \mathrm{dBm}$ and $n=0.8$ at the large $P_{\text {in }}$ of $-5 \mathrm{dBm}$.

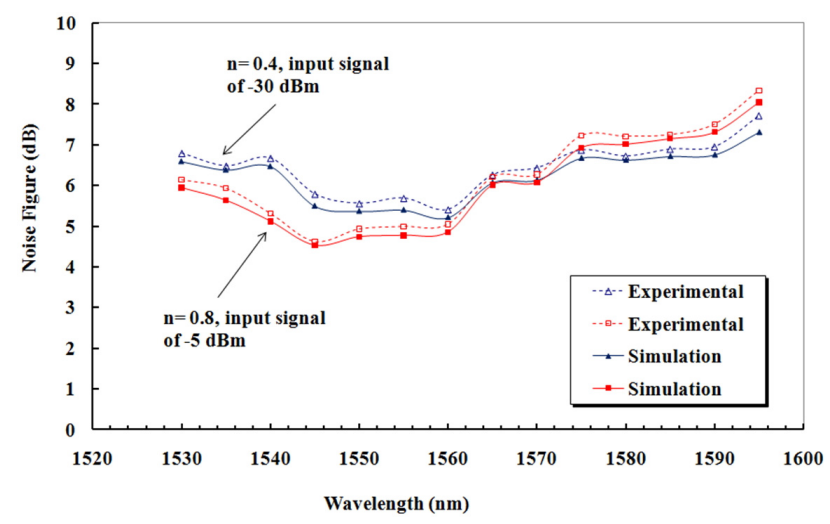

FIG. 6. Simulation \& experimental validation of hybrid noise figure for two coupling ratios of $n=0.4$ at the $P_{i n}$ of $-30 \mathrm{dBm}$ and $\mathrm{n}=0.8$ at the large $\mathrm{P}_{\text {in }}$ of $-5 \mathrm{dBm}$. signal and $9.9 \mathrm{~dB}$ for large signal. The gain variation is $1.06 \mathrm{~dB}$ at small signal and $0.77 \mathrm{~dB}$ at large signal for simulation while the values obtained from experiment are 1.07 and $0.81 \mathrm{~dB}$, respectively.

The simulated and experimental noise figure (NF) is shown in Fig. 6. The highest NF value of the small and large input signals in the $\mathrm{C}$-band region was recorded at $1530 \mathrm{~nm}$. This is because of the noisy amplifier spontaneous emission (ASE) at the short wavelength range. The worst $\mathrm{NF}$ in the L-band was observed at $1595 \mathrm{~nm}$ caused by steep gain drops, as it is quite far away $(15 \mathrm{~nm})$ from the maximum Raman gain at $1580 \mathrm{~nm}$.

\section{CONCLUSION}

The gain performance of a new gain-controlled P-HFA design is simulated and experimentally demonstrated. A wide gain bandwidth of $60 \mathrm{~nm}$ from $1530 \mathrm{~nm}$ to $1590 \mathrm{~nm}$ is obtained with input signal less than $-5 \mathrm{~dB}$. The gain variation is about $1.06 \mathrm{~dB}$ at small input signal. This variation is enhanced and reduced to $0.77 \mathrm{~dB}$ at the large input signal. The gain flatness is improved and the gain dynamic range is increased as well compared with the conventional P-HFA. Finally, the experimental results show good agreement with the simulation result.

\section{ACKNOWLEDGMENT}

The research is funded by Universiti Tenaga Nasional internal research grant with code J510050442 and the Research Council of the Sultanate of Oman under Research Grant Agreement No [ORG SU ICT11 002]. The authors also thank Yenista Optics Cooperation for equipment support.

\section{REFERENCES}

1. J. Yuan, T. Liang, W. Wang, and S. Gu, "Impact analysis on performance optimization of the hybrid amplifier (RA+EDFA)," Optik - Int. J. Light Electron Opt. 122, 1565-1568 (2011).

2. M. I. Azawe, "Low noise C-band EDFA/DRA hybrid amplifier using the same pump laser," Photonics Lett. Pol. 3, 165-167 (2011).

3. M. N. Guo, S.-K. Liaw, P. P. Shum, N.-K. Chen, H.-K. Hung, and C. Lin, "Single-wavelength-pump bi-directional hybrid fiber amplifier for bi-directional local area network application," Opt. Commun. 284, 573-578 (2011).

4. M. H. Abu Bakar, A. F. Abas, M. Mokhtar, H. Mohamad, and M. A. Mahdi, "Utilization of stimulated raman scattering as secondary pump on hybrid remotely-pump L-band Raman/erbium-doped fiber amplifier," Laser Phys. 21, $722-728$ (2011).

5. S. Singh and R. Kaler, "Flat-gain L-band Raman-EDFA hybrid optical amplifier for dense wavelength division 
multiplexed system," IEEE Photon. Technol. Lett. 25, 250-252 (2013).

6. M. H. Abu Bakar, F. R. Mahamd Adikan, N. H. Ibrahim, and M. A. Mahdi, "L-band R-EDFA/Raman hybrid amplifier with enhanced higher-order pumping scheme utilizing stimulated raman scattering," Opt. Commun. 291, 155-161 (2013).

7. M. Z. Jamaludin, F. Abdullah, M. H. Al-Mansoori, N. I. M. Rawi, S. M. Idris, and M. R. Haleem, "Remotely pumped hybrid double-pass L-band optical amplifier utilizing chirped fiber Bragg," Optik - Int. J. Light Electron Opt. 125, 620-623 (2014).

8. A. Carena, V. Curri, and P. Poggiolini, "On the optimization of hybrid Raman/erbium-doped fiber amplifiers," IEEE Photon. Technol. Lett. 13, 1170-1172 (2001).

9. S. Kawai, H. Masuda, K. Suzuki, and K. Aida, "Widebandwidth and long-distance WDM transmission using highly gain-flattened hybrid amplifier," IEEE Photon. Technol. Lett. 11, 886-888 (1999).

10. H. Masuda and S. Kawai, "Wide-band and gain-flattened hybrid fiber amplifier consisting of an EDFA and a multiwavelength pumped Raman amplifier," IEEE Photon. Technol. Lett. 11, 647-649 (1999).

11. J. H. Lee, Y. M. Chang, Y.-G. Han, S. H. Kim, H. Chung, and S. B. Lee, "Dispersion-compensating Raman/ EDFA hybrid amplifier recycling residual Raman pump for efficiency enhancement," IEEE Photon. Technol. Lett. 17, 43-45 (2005).

12. H. Masuda, S. Kawai, and K. Aida, "76-nm 3-dB gain-band hybrid fiber amplifier without gain-equalizer," Optical Amplifiers and Their Applications, 78-81 (1998).

13. S.-K. Liaw and Y.-S. Huang, "C+L-band hybrid amplifier using FBGs for dispersion compensation and power equalisation," Electron. Lett. 44, 3-4 (2008).
14. S. Liaw, K. Huang, W. Chen, Y. Hsiao, and I. Lai, "Investigate $\mathrm{C}+\mathrm{L}$ band EDFA/Raman amplifiers by using the same pump lasers," JCIS (2006), p. PNC-I I.

15. S. Padwal and M. Chattopaddhyay, "Performance analysis of hybrid optical amplifier in $\mathrm{C}$ and $\mathrm{L}$ band over EDFA and RFA," Int. J. Adv. Res. Electron. Commun. Eng. 2, 40-44 (2013).

16. S. K. Liaw, C. K. Huang, and Y. L. Hsiao, "Paralleltype C+ L band hybrid amplifier pumped by $1480 \mathrm{~nm}$ laser diodes," Laser Phys. Lett. 5, 543-546 (2008).

17. C. Giles and E. Desurvire, "Modeling erbium-doped fiber amplifiers," J. Lightwave Technol. 9, 271-283 (1991).

18. P. Becker, N. Olsson, and J. Simpson, Erbium-Doped Fiber Amplifiers Fundamentals and Technology (Elsevier, 1999).

19. M. K. Aseka and M. Menifb, "Protection of surviving channels in pump-controlled gain-locked Raman fibre amplifier," Opt. Commun. 210, 57-65 (2002).

20. C. Headley and G. Agrawal, Raman Amplification in Fiber Optical Communication Systems (Elsevier Academic Press, 2005).

21. B. Bristiel, P. Gallion, Y. Jaouën, E. Pincemin, F. T. R, T. Anticipa, and A. P. Marzin, "Intrinsic noise figure derivation for fiber Raman amplifiers from equivalent noise figure measurement," Proc. Lightwave Technologies in Instrumentation and Measurement Conference, 19-20 (2004).

22. R. H. Stolen and E. P. Ippen. "Raman gain in glass optical waveguides," Appl. Phys. Lett. 22, 276-278 (1973).

23. M. H. Ali, F. Abdullahi, M. Z. Jamaludini, M. H. AI-Mansoori, T. F. AI-Mashhadani, and A. K. Abass, "Effect of EDF position on the performance of hybrid dispersion-compensating Raman/EDF amplifier," in Proc. 4th International Conference in Photonics (ICP) (Melaka, Malaysia, 2013), pp. 187-189. 